UDC543.421/.424

\author{
${ }^{1}$ A.S. Drobyshev", \\ ${ }^{1}$ K.A. Katpayeva, ${ }^{2}$ Yu.M. Strzhmechny \\ ${ }^{1}$ Al-Farabi Kazakh National University, Almaty, Kazakhstan \\ ${ }^{2}$ Texas Christian University, Fort Worth, USA \\ "e-mail: Andrei.Drobyshev@kaznu.kz
}

\title{
Ir-spectrometric investigation on cluster composition of two-component solid films of nitrogen-ethanol
}

\begin{abstract}
Studies that were carried out previously investigating the properties of the cry condensed films of weak two-component solutions of ethanol in nitrogen found that the state of the film is not stable. A slight increase in temperature of solid nitrogen cryomatrix leads to changes in the vibrational spectrum of ethanol. This article is a continuation of this research and is aimed at studying the cluster composition of thin films of ethanol cry vacuum condensates in weak solutions with nitrogen. Measurements were carried out in a temperature range from 12 to $40 \mathrm{~K}$, the pressure in the vacuum chamber was less than $5 \times 10^{-8}$ Torr. The concentration of ethanol in nitrogen was varied in the range from $0.5 \%$ to $3 \%$.

Based on the analysis of the vibrational spectra, it is assumed that the two-component nitrogen-ethanol film contains clusters of various compositions, from dimers to polyaggregates. An increase in cryomatrixtemperature leads to transformations in these polyaggregates with formation of more stable forms, as demonstrated by changes in the fine structure of the considered bands. The presence in the spectra of the absorption bands, which frequencies correspond to the existence of ethanol dimers and monomers in nitrogen matrix, can be explained as follows. Part of the ethanol molecules forming polyaggregates may not be combined via hydrogen bonds with neighboring molecules, forming a broken chain. Thus, they form a set of quasi-free molecules with corresponding absorption bands of these states. It is assumed that the quasi-free unbound molecules are preferably arranged in the surface area of the clusters.

Key words: cry matrix, polyaggregate, hydrogen bond, polymorphic, cry condensate.
\end{abstract}

\section{Introduction}

One of the interesting problems of modern solid state physics is providing the fundamentals and developing mechanisms of the formation of nanoobjects [1]. Extremely effective method for studying the formation and properties of nanoobjects is a method of cryomatrix isolation. The basis of this method [2] is the process of cocondensation with the matrix gas vapors or gases analytes lifetimes of which for various reasons is very limited under normal conditions. Low temperature of the substrate up to $\mathrm{T}=4 \mathrm{~K}$, restricts reactivity of the components, so as a result thin film is composed of a matrix with embedded active elements, which at a given temperature does not come into contact with the adjacent elements and the matrix itself. Cryomatrix isolation method proved to be particularly effective in the study of cluster formation processes in single-component and multicomponent systems. Under these conditions, the inert matrix and ultra-low temperature provides useful information about the properties of atoms and molecules in an unrelated, quasi-free state.

In the last decades it is observed the shift of accent of this method's application from mainly analytical to its more and more technological use. It is caused in essential degree by parallel development as nanosciences as nanotechnologies. It is known that the nanoobjects can be obtained in two principally different ways - from «above» and from «below», i.e. by destruction of macroobjects before obtaining nanoscale formations (a way "from above"), or assembly of nanoobjects directly from atoms and molecules (a way "from below"). And so, the method of cryomatrix isolation refers to one of the most effective methods of nanosystems 
formation "from below" allowing forming nanosystems with predetermined properties consciously and purposefully.

Specified practical value of the cryomatrix isolation method requires significant expansion of concentration ratios parameters in the system of cryomatrix-studied object. In conventional studies of fundamental nature these ratios between agentmatrix range from $1 / 1000$ to $1 / 100000$ [4], depending on the activity of monomers and conditions of the problem. It is understood that the accumulation of final amount of the substance can be a very time consuming process. Investigation on the behavior of matrix-isolated systems with a sufficiently high concentration of the analyte in the matrix became relevant due to and with regard to the requirements of applied nanophysics. These ratios may reach values of $1 / 100$ to $1 / 20$ or more. Thereat the concept itself of "cryomatrix isolation" is interpreted in a broader sense. In fact, it is a kind of solid solution when the molecules of the studied compound somehow embedded in a matrix (in other words, in the crystal structure) of another substance.

The present work is devoted to studying the processes of formation and properties of the ethanol clusters which are formed by method of cryomatrix isolation in the course of a co-condensation of a gas phase of ethanol and nitrogen on the cooled metal substrate. The choice of the studied substance (ethanol) is caused by existence of hydrogen bond between ethanol molecules that promotes formation of different structural states and thermo stimulated transformations between these states. IR spectrometry method of analysis is selected as the main tool for studying the composition of the films.

\section{Results and discussion}

The measurements were performed on a modified installation described in detail in [5]. It also includes a description of the sequence of experiments implemented in these studies, virtually unchanged. Technique of obtaining information about the water molecules in the matrix implies the analysis of the amplitude of nitrogen absorption band corresponding to fluctuations of ethanol unbounded molecules.

The analysis of thin structure of an absorption band of O-N bond of ethanol in the range of 3000$3600 \mathrm{~cm}^{-1}$ is given in figure 1 . The presented data indicate an existence of the local minima corresponding to various cluster conditions of ethanol molecules in a nitrogen matrix. Based on calculations and experimental data of other authors [6-8], we draw a conclusion about ethanol clusters of various size existences in a matrix of nitrogen. The frequencies of local minima and their interpretation according to the data provided in [9], are shown in the figure. Thus the following contracted notations are accepted: m-monomer, ddimer, cycl. d-cyclic dimer, cycl. trim. - the cyclic trimmer, cycl. tetr. - cyclic tetramer, hex-hexomer. Besides, an appearance of a wide strip in the range of 3250-3330 $\mathrm{cm}^{-1}$ might mean an appearance in a matrix of large polyaggregates, in which ethanol molecules are in a hydrogen bounded state appearance - they are designated as multimer.

It should be noted that in the publications quoted by us units, which sizes are more than dimer, are mainly cyclic. This property of ethanol molecules to close hydrogen bonds cyclically, forming the steadiest cluster structures, can appear the fundamental reason of ethanol ability to form steady polymorphic and polyamorphous, including vitreous, states.

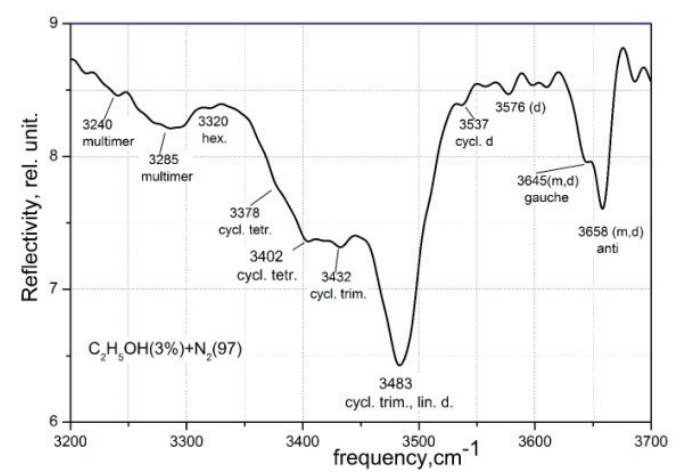

Figure 1 - Features of the absorption band of the O-H bond of ethanol (3\%) in a nitrogen cryomatrix (97\%). Notation: (m) monomer, (d) dimer, (cycl. d) cyclical dimer, (cycl. trim.) cyclical trimer, (cycl. tetr.) cyclical tetramer, (hex.) hexamer.

Figure 2 shows similar results for the frequency range of the bending vibrations.

Consider in more detail the frequency range $1200-1500 \mathrm{~cm}^{-1}$, which includes the bending vibrations of different bonds of ethanol molecules, or there combinations with rotational vibrations. Figure 2 shows the fine structure of the spectrum at the selected frequency range in a mixture of ethanol and nitrogen (3\% and 97\%) - upper graph and the spectrum of pure ethanol is shown for comparing in the same range. 

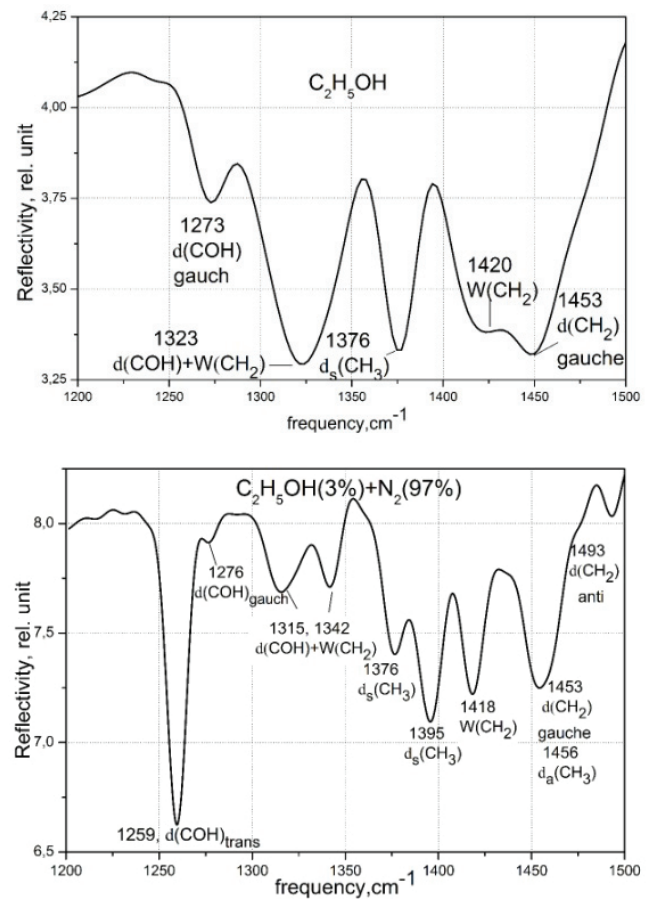

Figure 2- The characteristic frequencies of vibrations of mixture molecules with $3 \%$ of ethanol and $97 \%$ of nitrogen (lower graph) and cryocondensate of $100 \%$ pure ethanol (upper graph).

Obviously, the spectrum of ethanol - nitrogen mixture in concentration ratio $(97 \%+3 \%)$, has a much more complex fine structure in comparison with the vibrational spectrum of $100 \%$ concentration ethanol. 9 peaks are clearly defined at the spectrum of the mixture in the frequency range $1200-1500 \mathrm{~cm}^{-1}$, whereas the spectrum of pure ethanol shows only five of them. Let us try to analyze these important differences. Band with a maximum at frequency of $1493 \mathrm{~cm}^{-1}$ corresponding to the bending vibrations of the $\mathrm{CH}$ bond of $\mathrm{CH}_{2}$ group of ethanol anti-conformers, completely disappears in the spectrum of pure ethanol. The band of symmetric bending vibrations of $\mathrm{CH}_{3}$ group at a frequency $v=1376 \mathrm{~cm}-1$ in the spectrum ofethanol and nitrogen mixture divided into two bands, which frequency maxima are at 1376 and $1395 \mathrm{~cm}^{-1}$. The situation is similar in the case of a combination of bending vibrations of $\mathrm{COH}-$ bond and rotational vibrations of the $\mathrm{CH}$ bond of methylene group. One band in the spectrum of pure ethanol at a frequency $v=1323 \mathrm{~cm}^{-1}$ is divided into two bands with centeres at frequencies of 1315 and $1342 \mathrm{~cm}^{-1}$.

Of particular interest is the absorption band of bending vibrations of the COH-bond for the anti and gauche conformers at frequencies 1259 and $1276 \mathrm{~cm}^{-1}$. The band of ethanol anti conformer having a frequency of $1259 \mathrm{~cm}^{-1}$ is completely missing in the spectrum of pure ethanol, whereas the band of gauche conformal vibrations not only retained, but in this case becomes pronounced. The reason for this behavior of ethanol spectra lies in $\mathrm{COH}-$ bond anti conformers participation in formation between ethanol molecules isolated in a matrix of nitrogen, of hydrogen bonds, by which the molecule subsequently combined into cyclic clusters, then this type of vibrations is braked. Thus, on the basis of consideration of this band we can make the following conclusion. Immutability of the behavior of vibrational spectrum of $\mathrm{COH}$-bond gauche conformers of ethanol vibrations suggests that the $\mathrm{COH}$-bond of gauche-isomer not involved in the formation of clusters in the system «matrixethanol».

On the basis of the analysis of the obtained IRspectra it is possible to draw a conclusion on ethanol monomers and dimer presence in a matrix of nitrogen. It is indicated by the absorption band at a frequency of $3658 \mathrm{~cm}^{-1}$ caused by $\mathrm{O}-\mathrm{H}$ bond fluctuations of ethanol monomers and dimers. Existence of local minima at frequencies of 3645 and $3658 \mathrm{~cm}^{-1}$ at this strip is connected with the existence of ethanol molecule two conformational states, anti $\left(3658 \mathrm{~cm}^{-1}\right)$ and gauche $\left(3645 \mathrm{~cm}^{-1}\right)$.

Local minima in the range of $3000-3600 \mathrm{~cm}^{-1}$ indicate also an existence in a matrix of the following units of ethanol: m-monomer, d-dimer, cycl. d-cyclic dimer, cycl. trim. - the cyclic trimmer, cycl. tetr. - cyclic tetramer, hex-hexomer. The wide band in the range of $3250-3330 \mathrm{~cm}^{-1}$ means an existence in a matrix of large polyaggregates, in which molecules of ethanol are in a hydrogen bounded state (multimer).

\section{References}

1. Guanzhong Cao. Nanostructures and Nanomaterials: Synthesis, Properties and Applications. World Scientific Publishing Co. Pte. Ltd. Singapore, 2004. - 448 p.

2. Antony J. Tursi, Eugene R. Nixon. MatrixIsolation Study of the Water Dimer Nitrogen // Chemical Physics. - 1970. - Vol.52. -N. 3. - P. 1521-1528.

3. Cao G., Wang Y. Nanostructures and Nanomaterials: Synthesis, Properties and Applications. World Scientific Publishing Co. Pte. Ltd. - Singapore, 2011. - P. 369-422. 
4. Surovtsev N.V, Adichtchev1 S.V., E R“ossler1 and Ramos M.A. Density of vibrational states and light-scattering coupling coefficient in the structural glass and glassy crystal of ethanol // Journal of Physics: Condensed matter. - 2004. Vol. 16. - P. 223-230.

5. Drobyshev A., Abdykalykov K., Aldiyarov A., Kurnosov V., Tokmoldin N. IR spectra of water polyaggregates in a nitrigoncryomatrix // Low temperature physics. - 2007. - Vol.33. - N. 8. P.699-703.

6. Wasserman T., Suhm M. Ethanol Monomers and Dimers Revisited: A Raman Study of Conformation Prefences and Argon Nanocoating
Effects // Journal of Chemical Physics. - 2010. Vol. 114. - P. 8223.

7. Zielke and M. Suhm. Concerted proton motion in hydrogen-bonded trimers: A spontaneous Raman scattering perspective // Journal Physical Chemistry // Chemical Physics. -2006.- N. 8. - P.2826-2830.

8. W.A.P. Luck, Schrems O. Infrared Matrix Isolation Studies of Selfassociation of Methanol and Ethanol // Journal of Molecular Structure. 1982. - Vol. 60. - P.333.

9. Gonzalez M.A., Enciso E., Bermejo F.J., Bee M. Hydrogen bonding in condensed-phase alcohols: some keys to understanding their structure and dynamics // Physical Review B. - 2000. Vol.61. - P. 6654. 